\title{
Philosophiques
}

\section{Société et politique avec ou sans État}

\section{Joseph Pestieau}

Volume 6, numéro 2, octobre 1979

URI : https://id.erudit.org/iderudit/203117ar

DOI : https://doi.org/10.7202/203117ar

Aller au sommaire du numéro

Éditeur(s)

Société de philosophie du Québec

ISSN

0316-2923 (imprimé)

1492-1391 (numérique)

Découvrir la revue

Citer cet article

Pestieau, J. (1979). Société et politique avec ou sans État. Philosophiques, 6(2), 235-252. https://doi.org/10.7202/203117ar d'utilisation que vous pouvez consulter en ligne.

https://apropos.erudit.org/fr/usagers/politique-dutilisation/ 


\title{
SOCIÉTÉ ET POLITIQUE AVEC OU SANS ÉTAT
}

\author{
par Joseph Pestieau
}

Je tâcherai tout d'abord de définir ce qu'est la politique au sein d'une société et ce qu'est l'État au sein de la politique. Je montrerai ensuite que, s'il n'y a pas de société sans politique, il y a des sociétés sans État. Celles-ci refusent tout pouvoir qui serait séparé d'elles-mêmes, mais elles n'en sont pas moins soumises à un ordre idéologique incontestable. Leur conservatisme est absolu, quoiqu'il ne soit pas sans raison. En tout cas, ces sociétés ne réalisent ni l'idéal libertaire ni l'idéal anarchiste.

\section{Politique et société}

Sous ce titre, il sera question de la nécessité d'un ordre politique dans toute société, que cet ordre soit assuré par une tradition immémoriale ou par la contrainte du plus fort. Il s'agira de circonscrire la fonction du politique de la façon la plus générale qui soit, sans la lier nécessairement à l'État.

C'est au sein d'une société que les individus s'établissent, s'identifient, gagnent éventuellement en puissance, se valorisent comme ils peuvent et trouvent une sécurité relative. Ils peuvent se contenter des relations sociales qu'ils entretiennent les uns avec les autres. Il arrive aussi qu'ils discutent et luttent pour établir des relations qui leur conviendraient davantage, qu'ils protègent celles qui leur conviennent déjà. S'ils se préoccupent de l'organisation sociale dans son ensemble et des institutions, des lois et des coutumes qui y correspondent, ils font de la politique, dit-on. Ce peut être par intérêt, par idéal ou parce qu'ils aiment le pouvoir et trouvent un champ d'action à la mesure de leurs ambitions dans l'exercice d'une influence aussi large. Habituellement, ces trois motifs se 
mêlent intimement. Ainsi, des intérêts communs ne réunissent un parti que sous la direction de chefs qui ont quelque ambition, et il faut que ces intérêts communs puissent passer pour le bien commun afin de paraître légitimes. Qui départagera la part des idéaux, celle des intérêts et celle de l'ambition?

Les conflits d'intérêts, d'idéaux ou d'ambitions provoquent la cristallisation de solidarités et de passions. Bien des individus s'identifient à une solidarité ou à une passion et prennent parti avant de savoir clairement leurs intérêts et leurs idéaux, les ambitions qu'ils servent ou celles qu'ils poursuivent. Des intérêts déterminent sans doute les antagonismes politiques, mais ceux-ci s'instituent et durent au-delà des causes qui les avaient suscités. La distribution des partis ou des factions s'entretient par la volonté de leur appareil et l'habitude de leurs clients. Les batailles politiques peuvent ne plus correspondre à des enjeux importants. Elles n'en auront pas nécessairement moins de virulence. Il se peut que, pour mieux oublier l'insignifiance et la stérilité de ces batailles, on y investisse toutes ses énergies. Les clivages, en politique intérieure comme en politique extérieure, peuvent être entretenus pour eux-mêmes, parce qu'ils offrent à beaucoup de monde un des rares moyens de jouer un rôle grandiloquent.

La politique concerne l'ensemble de l'organisation sociale. Mais tout dans cette organisation ne relève pas également de la politique. Celle-ci concerne cet aspect de l'organisation sociale qui est sanctionné par la force, une force qui s'impose en droit, constitue une dernière instance et donc une garantie de paix. La politique assure un ordre intérieur et une protection contre l'extérieur, elle structure, encadre, protège l'organisation sociale mais ne se confond pas avec celle-ci. L'État, et plus particulièrement la police, l'armée ou l'administration qui exécutent la volonté de l'État, ou l'administration qui exécute ses décisions, sont souvent ressentis par la société comme des contraintes extérieures. Démocratie ou pas, ce sont des corps spécialisés, dont la fonction est d'imposer des règles au sein d'une sociétél.

1. On peut faire une distinction entre deux sens des mots "organisation politique ": 1) l'organisation sociale en tant que structurée politiquement et 2) le corps spécialisé qui 
Il y a cependant des sociétés sans État, sans force armée, où le pouvoir politique est diffus, non explicité, jamais monopolisé par des personnes ou des organisations spécialisées. Par exemple, tous les adultes mâles ou tous ceux d'un lignage particulier ou toute une classe d'âge peuvent y participer. Dans de tels cas, l'organisation politique n'est pas prise en charge par un corps constitué qui se distinguerait clairement au sein de l'organisation sociale. Les chasseurs-cueilleurs du Kalahari ou de la forêt amazonienne ne s'accommodent que de chefs sans autorité, qui tâchent de dire la coutume, de parler au nom du groupe, d'amener des partis opposés à un compromis. Ils ne peuvent rien imposer, ils n'ont pas de pouvoir. Mais alors doit exister, au moins dans les communautés locales et dans la sphère des activités usuelles, un consensus au sujet des droits et des devoirs de chacun. Quant aux dérogations aux coutumes, elles peuvent être réprimées par le ridicule ou par des menaces mystiques aussi sûrement que par la force armée ${ }^{2}$.

L'unanimité et l'absence de corps policier qu'on trouve dans certaines cultures reposent sur la répétition de modèles ancestraux. Une forte ritualisation des rôles et des devoirs les situe au-delà du discutable ${ }^{3}$. Ces cultures sont donc soumises à un ordre politique, contraignant comme n'importe quel ordre politique, et incontestable de surcroit. Qu'on n'y trouve pas de division en classes, que l'exploitation de l'homme par l'homme y soit réduite, que cet ordre soit meilleur que le nôtre, ce sont d'autres questions. Il me semble qu'il faut une certaine spécialisation ou personnification des rôles politiques pour que les hommes puissent se distancer de l'ordre qui les régit. Si la loi d'un maître écrase, au moins peut-on la reconnaître, la critiquer et la renverser ${ }^{4}$.

assume la fonction politique. Cf. David EASTON, "Political Anthropology ", in Biennial Revieu of Antbropology, 1959, édité par B. Siegel, Stanford University Press, Stanford, 1959.

2. Cf., à ce propos, la préface de A. R. RADCLIFFE-BROWN et l'introduction de M. FORTES \& E. E. EVANS-PRITCHARD in Systèmes politiques africains, édité par Fortes et Evans-Pritchard, P.U.F., Paris, 1964. Cf. également l'article d'EASTON déjà cité et $\mathrm{G}$. BALANDIER, Antbropologie politique, P.U.F., Paris, 1967.

3. Cf. Essays on the Ritual of Social Relations, édité par Max Gluckman, Manchester University Press, Manchester, 1962.

4. Les grandes transformations délibérées qui font l'histoire semblent n'apparaître qu'avec la contrainte étatique. Serait-ce parce quil faut une telle contrainte pour imposer de telles transformations? Ne serait-ce pas aussi parce que la contestation dont la contrainte 
Dans bien des sociétés africaines, la contestation permanente des maîtres et la concurrence des allégeances politiques n'abolissent pas la continuité de l'ordre politique. Différents groupes peuvent tâcher de faire prévaloir des principes de solidarité bien distincts. Et pourtant entre ces principes, un équilibre se rétablit sans cesse. Jamais il ne s'éloigne trop d'un modèle coutumier. La contestation des rois ou des chefs aboutit tout au plus au remplacement du personnel politique dans les mêmes cadres. La plupart du temps, elle permet à des tensions de se résorber en s'exprimant de façon dramatique, et le statu quo est sauf. Même quand une nouvelle unité politique se crée, par fission ou fusion, c'est un même régime qui dure, ce sont les mêmes rôles et statuts qui sont redistribués. Souvent la religion est invoquée pour rappeler et imposer un minimum d'ordre - celui qui est conforme au vouloir des ancêtres, à la bonne intelligence des morts et des vivants, à l'ordre des choses, à la volonté des dieux - quand les conflits entre factions rivales semblent ne laisser subsister aucune instance capable de discipliner ces conflits ${ }^{5}$. D'ailleurs, la faction qui réussit à se porter à la défense ou à s'associer à la célébration de l'ordre coutumier marque des points contre les autres. En se mettant au service d'une loi qui prime sur toutes les autres, elle gagne en légitimité, elle augmente le nombre de ses partisans ou réduit celui de ses opposants ${ }^{6}$. En même temps qu'elle proclame cette loi, qui prime toutes les autres, et lui donne une nouvelle actualité, elle la manipule aussi à son avantage. Si la coutume ne se perpétue que parce qu'elle sert les puissants, ces derniers ne deviennent puissants que parce qu'ils ont su se servir de la coutume.

Sous les apparences d'un grand désordre, un certain ordre politique peut donc perdurer. Même s'il y a plusieurs chefs ou plusieurs principes de solidarité en concurrence, un modus

étatique fait l'objet provoque de telles transformations? En d'autres mots, l'histoire apparaîtrait avec la différenciation sociale, avec la lutte pour le pouvoir et l'émergence de classes sociales.

5. C'est la thèse de Victor TURNER in Schism and Continuity in an African Society, Manchester University Press, Manchester, 1957.

6. Cf. V. TURNER, "Ritual Aspects of Conflict Control in African Micropolitics», in Political Antbropology, édité par Schwartz, Turner \& Tuden, Adline Publishing Co., Chicago, 1966, p. 236-246. 
vivendi peut s'établir entre eux. Il y a des régimes féodaux en équilibre même si chaque palier de gouvernement prétend à la souveraineté. Les peuples s'accommodent de l'instabilité de leurs maîtres pour autant que leur droit et leur coutume soient respectés et qu'un minimum de sécurité soit sauvegardé. Mais si l'instabilité et l'insécurité portent sur la vie quotidienne, alors la situation est insupportable. Un peuple peut alors choisir de soutenir la cause de qui lui offre sécurité et se porte garant de l'ordre. C'est ainsi que le mieux armé peut gagner une légitimité. Dans les désordres de la guerre de Cent ans, la France découvrit les vertus d'un roi et d'un État. Le Royaume d'Angleterre sortit plus fort de la guerre des Deux-Roses parce que le peuple aspirait à la paix, et non seulement parce que les grands nobles étaient affaiblis ${ }^{7}$. Des tribus entières ont bien accueilli la garantie d'ordre que leur offrait le régime colonial parce que l'administration indirecte que pratiquait ce régime sauvegardait apparemment leur coutume et la paix. Dans le Sertao brésilien, au début du siècle, on a vu des bandes de hors-la-loi faire la loi. La population et même les fonctionnaires se mettaient sous leur protection quand ils ne pouvaient plus attendre une protection efficace de la police de l'État. Il valait mieux faire allégeance aux bandits que d'être pris entre le feu de ceux-ci et le feu de la police ${ }^{8}$.

On ne peut donc vivre sans un ordre défini. Il faut bien qu'une règle s'impose. En ce sens, il n'y a pas de société sans politique. Mais certaines sociétés s'accommodent d'un pouvoir décentralisé ou même très diffus et de ce qui apparaît à d'autres un désordre intolérable. Certaines sociétés n'ont pas de maîtres mais elles n'en sont pas moins soumises à un ordre moral. Dans ce cas, il serait bien difficile de distinguer entre organisation politique et organisation sociale.

7. Nation ou peuple, évidemment, ne rassemble pas des individus égaux. Les intérêts qui soutiennent la cause du roi en France après la guerre de Cent ans, la cause du roi d'Angleterre après la guerre des Deux-Roses, sont des intérêts importants mais non les intérêts de tous.

8. D'ailleurs la police ne se conduisait pas mieux que les bandits. Cf. M. I. PEREIRA de QUEIROZ, Os Cangaceiros, Julliard, Paris, 1968. 


\section{2. État et société}

L'État se présente comme pouvoir souverain. En fait, il se fonde sur des rapports de forces mouvants et des compromis plus ou moins habiles. Il n'est pas indépendant des luttes sociales qu'il prétend ordonner. C'est ce qu'il faut saisir pour comprendre ce qu'est l'État.

Celui-ci tend à monopoliser la force armée et la fiscalité, les gens d'armes assurant les recettes fiscales et celles-ci assurant la paie des gens d'armes. La centralisation qui caractérise l'État suppose qu'il se soit imposé aux pouvoirs rivaux ou périphériques et que ses serviteurs ne deviennent pas à leur tour des princes locaux plus ou moins autonomes. La féodalisation est inévitable là où le pouvoir central, faute de liquidités, de moyens de contrôle ou de communication, ne peut établir ses agents qu'en leur accordant des fiefs qui deviennent rapidement une base de pouvoir indépendant. Il y a néanmoins bien des formes intermédiaires entre un État centralisé et la dissolution de toute autorité étatique dans l'émiettement des fiefs. Ou bien des princes locaux peuvent reprendre à leur compte l'autorité de l'État, s'imposer comme souverains dans leur territoire et tâcher d'élargir celui-ci. Ou bien un pouvoir central subsiste en composant avec des féodaux, et ceux-ci gardent une certaine autonomie en composant avec le pouvoir central. D'autre part, dans un État dont l'unité et la souveraineté sont bien établies, la compétition pour le pouvoir ne cesse pas, mais les antagonistes visent moins à remettre en cause la centralisation qu'à l'utiliser à leur profit.

Selon la perspective marxiste, l'État apparâ̂trait avec la différenciation des classes sociales. Il serait un instrument par lequel une classe consoliderait sa domination sur les autres. Il assurerait l'intégration d'une société ${ }^{9}$ que divisent des intérêts divergents. Avec toute la force dont il est seul capable, il garantirait le bon fonctionnement d'un système de production qui oppose des exploiteurs et des exploités. Cependant, les

9. Si la société n'est unie que pour le bénéfice d'une classe particulière et par la force d'une police ou d'une propagande, certains préferent parler de formation sociale plutôt que de sociécé, ce dernier mot évoquant une unité spontanée. 
différentes classes se transforment en même temps que les intérêts qui les unissent ou les désunissent. Sur les bases de l'exploitation, dans les cadres que l'État donne à cette exploitation, les forces productives peuvent se développer rapidement ${ }^{10}$, de nouvelles classes émergent et gagnent en importance. Il faut alors comprendre comment le rapport des forces sociales - et des classes qui correspondent à ces forces - s'exprime politiquement et s'établit, notamment à l'occasion des luttes pour le pouvoir politique. Car la façon dont les forces sociales changent, s'allient ou s'opposent, prennent conscience d'elles-mêmes et font évoluer les idéologies, transforment les institutions ou s'en accommodent et, finalement, constituent un système de pouvoir, n'est guère prévisible. C'est ce qui fonde l'originalité du point de vue politique au sein même de la théorie marxiste ${ }^{11}$.

L'État prétend régir les cadres généraux d'une société délimitée, celle qui correspond à une aire où il peut exercer un monopole ou un quasi-monopole de la force armée pour soutenir son administration. Il représente une dernière instance plus nettement que ne pouvait le faire toute autre forme de pouvoir politique ${ }^{12}$. On comprend l'importance et l'âpreté des conflits pour accaparer ou influencer un tel pouvoir qui, par définition, se présente comme souverain. Mais il n'est jamais aussi énorme qu'une telle définition le laisse entendre.

Ce pouvoir ne porte que sur des cadres généraux. Il doit bien se limiter à ce qu'il peut contrôler effectivement. Même avec une volonté totalitaire, une administration centralisée et bien rodée, une police efficace, le pouvoir politique ne pourrait tout superviser. Il coexiste avec des initiatives qui lui

10. Cf. ENGELS, Anti-Diibring (traduction de Bottigelli), Les Éditions sociales, Paris, $3^{e}$ édition revue, 1971 , p. 208 et suivantes.

11. Cf., à ce sujet, Nicos POULANTZAS, L'État, le pouvoir, le socialisme, P.U.F., Paris, 1978 ou Marc AUGÉ, Pouvoirs de vie et pouvoirs de mort, Flammarion, Paris, 1977, p. 90-96 sous le titre " Rapports des forces et rapports de pouvoir ". Cf. surtout Le 18 brumaire de Louis Bonaparte et Les Luttes de classes en France (1848-1850) de MARX. L'État n'est pas seulement un enjeu des luttes politiques. Il est aussi un des lieux de ces luttes. Alors même que l'État prétend être au-dessus des intérêts de classe et des conflits sociaux, il est un théâtre où s'expriment ces intérêts et ces conflits. En d'autres mots, la domination d'une classe sur les autres par l'État n'est pas assurée une fois pour toutes.

12. Aussi est-ce souvent à partir de la forme étatique que l'on définit le pouvoir politique, comme si cette forme en était l'idéal-type. 
échappent plus ou moins. Il s'appuie à l'occasion sur elles comme à l'occasion elles s'appuient sur lui. Il tolère souvent, parce qu'il est plus pratique de les tolérer, des infractions aux règles mêmes qu'il a définies. Ce peut être par fatigue ou pour ne pas affronter plus fort que lui et devoir avouer sa faiblesse. Ce peut être aussi pour des raisons d'économie : une sanction exemplaire de temps en temps suffit souvent à maintenir un minimum d'ordre et d'autorité. Sans doute, tout pouvoir s'emploie à mieux s'établir mais, pour s'établir et durer, il lui faut tenir compte de ce qui pourrait le contester. Ainsi bien des souverains ou des premiers ministres ne le demeurent que parce qu'ils acceptent de laisser beaucoup d'autonomie à des Églises, à des fiefs administratifs, à des régions entières, à ce qui porte le nom de multinationales ou à des espèces de mafia.

Si le pouvoir de l'État paraît sans partage, c'est qu'il respecte le rapport des forces sociales, sert celles qui prédominent, mais évite aussi de provoquer la révolte des autres. Il ne sert d'ailleurs les premières et n'assure l'ordre qui leur convient qu'en se faisant passer pour l'arbitre ou le maître équitable de toutes, le garant de la paix, de la justice ou du développement. Ce rôle amène l'État à être un peu plus qu'un simple instrument de la classe dominante, à tenir compte des revendications les plus criantes de diverses classes, à y chercher des alliés. Comme toute institution, il tend vers une certaine autonomie, et ce d'autant plus que son rôle s'accroît. Cependant, même si l'État réussit à diversifier ses appuis et à gagner ainsi une certaine indépendance, il continue à dépendre de tous ceux sur qui il s'appuie.

Des princes firent prévaloir l'État national contre les féodaux en ménageant davantage le Tiers État, en s'appuyant sur le haut clergé, en arbitrant les conflits en faveur de ceux qui étaient susceptibles de leur demeurer fidèles. Ce que je dis ici à propos des limites du pouvoir de l'État moderne vaut aussi pour d'autres formes d'organisation politique. La suprématie d'un chef de guerre sur toute une région peut dépendre des droits qu'il a su garantir, des privilèges qu'il a accordés judicieusement, des alliés qu'il a honorés, autant que de ses conquêtes. 


\section{Société contre État}

Sous ce titre, je tâcherai d'abord de définir les sociétés sans État comme refus du pouvoir, de la différenciation sociale et de tout changement aux coutumes héritées. C'est en conservant celles-ci et en les respectant intégralement que des hommes peuvent coexister sans maîtres. J'envisagerai ensuite le cas de quelques cultures où il y a des mâttres qui n'ont que les apparences du pouvoir. Enfin, je discuterai du rôle primordial de l'idéologie dans le maintien des sociétés sans État et dans l'émergence des sociétés étatiques.

Jusqu'ici, j’ai plutôt insisté sur la continuité entre ces deux types de société. C'est qu'il s'agissait de cerner la fonction politique présente dans l'une et l'autre. Mais il faut aussi parler de leur dissemblance. On peut reprendre pour ce faire, quitte à le nuancer ensuite, le point de vue marxiste qui fait de l'État un aspect de la différenciation des classes sociales. L'État, c'est le pouvoir des uns sur les autres, le pouvoir des uns au détriment des autres, quoique les premiers puissent prétendre et réussissent à faire croire que le pouvoir de l'État, leur pouvoir, est au bénéfice de tous. On peut parler d'État même quand il est partagé entre un suzerain et des vassaux, même quand il est l'enjeu d'une contestation permanente entre puissances rivales, car ce qui compte dans le phénomène étatique c'est la contrainte organisée que peuvent faire peser sur les autres ceux qui ont réussi à établir un mode de production qui fonctionne à leur avantage.

Une société sans État, par contre, ne connaît pas la division en classes ni l'exploitation d'une classe par une autre. Cela n'empêche pas d'autres formes d'exploitation, celle de la femme par l'homme à l'intérieur de la famille, celle des cadets par les aînés à l'intérieur d'un lignage, par exemple ${ }^{13}$. Cependant, une telle société semble caractérisée par une impossibilité technique ou par un refus politique de l'accumu-

13. Cf. notamment Claude MEILLASSOUX, Femmes, greniers et capitaux, François Maspero, Paris, 1975, p. 116 et suivantes. Cf. surtout Mary DOUGLAS, The Lele of the Kasai, published for the International African Institute by the Oxford University Press, Londres, 1963, notamment p. 223. Il faut éviter de transformer les sociétés sans État en un repoussoir idéal de notre propre société. 
lation privée de biens de production et même de tout bien. On y décèle non seulement une absence de spécialisation du travail, mais aussi un contrôle du corps social sur l'ascendant que pourrait prendre certains spécialistes du fait de leur monopole (le forgeron, par exemple, ou le chef gardien des greniers communautaires). L'égalité ne s'entretient que dans un sous-développement relatif de l'appareil de production ${ }^{14}$. Mais cela n'implique pas nécessairement que l'on n'ait que la pénurie à partager ${ }^{15}$. On peut employer la notion d'économie de subsistance à propos des sociétés sans État,

" dès lors que l'on entend par là non point la nécessité d'un défaut, d'une incapacité inhérents à ce type de société et à leur technologie, mais au contraire le refus d'un excès inutile, la volonté d'accorder l'activité productrice à la satisfaction des besoins. Et rien de plus ${ }^{16}$. "

D'autre part, ce type de société non seulement valorise beaucoup le loisir et fort peu le travail, mais encore il s'avère capable de produire des surplus en vue de fêtes, de beuveries, d'invitations et de consommations communautaires ${ }^{17}$.

Pour reprendre un langage classique en philosophie politique depuis Hobbes, on peut définir la société sans État comme liée par un contrat social unissant chacun de ses membres à tous les autres, mais ne les soumettant pas à un pouvoir qui serait détaché d'eux. Pas de contrat avec un Léviathan, mais un contrat entre semblables qui entendent le demeurer ${ }^{18}$. Un contrat qui réalise l'union sans passer par la soumission et l'obéissance à un maitre. Mais ce contrat n'a rien de libéral pour autant. Car les membres individuels de la communauté se trouvent fondus dans celle-ci et, comme je le signalais plus haut, quelle autonomie peuvent avoir les individus soumis à un ordre culturel qu'ils ne peuvent mettre à

14. Ou pour dire les choses autrement : c'est la compérition sociale qui semble à l'origine de la production de surplus et du développement des forces productives. Cf. Maurice GODELIER, Horizon. Trajets marxistes en antbropologie, Maspero, Paris, 1973, p. 152. Produire plus que ses besoins, c'est produire pour un maître ou pour se préparer des dépendants.

15. Cf. Marshall SAHLINS, Age de pierre, agge d'abondance, Gallimard, Paris, 1976, notamment le premier chapitre.

16. Pierre CLASTRES, La Société contre l'État, Éditions de Minuit, Paris, 1974, p. 166.

17. Ibid., p. 166-167.

18. Cf. Bertrand de JOUVENEL, Du Pouvoir, Hachetre, Paris, édition 1977, p. 71-75. 
distance d'eux-mêmes ni combattre comme une contrainte extérieure ${ }^{19}$, qu'ils ne peuvent même pas objectiver?

Toute société suppose un ordre des moeurs, et celui-ci doit bien s'imposer, que ce soit par la force des armes ou celle de la seule coutume, coutume en accord avec la volonté des dieux. Que l'autorité qui régit les moeurs soit anonyme, tradition immémoriale hors de l'atteinte des mortels, tous égaux dans la soumission, ou qu'elle soit le vouloir d'un mortel qui a réussi à s'imposer aux autres, peu importe ${ }^{20}$. Il est de sa nature d'échapper aux prises du commun.

Dans une société sans État, l'ordre culturel est trop nécessaire pour être mis en cause par des discussions et des tergiversations. Même si en pratique il est souvent réaménagé, on fait comme s'il était de toute éternité. C'est ainsi qu'on s'oblige et qu'on se lie au respect des règles, qu'on assure la stabilité, qu'on renonce à la fois au désordre et à la liberté. Car on les craint plus encore qu'on ne les désire, on les craint d'abord quitte à les désirer ensuite avec nostalgie. Encore faut-il remarquer qu'il y a des cultures moins timides les unes que les autres vis-à-vis de la liberté et du désordre.

Une société sans État a bien des porte-parole et des leaders qui la conduisent, mais toujours selon la coutume. Leur rôle est limité et sous le contrôle de tous. Leur parole ne commande pas, mais rappelle la loi ancestrale et exprime la

19. Cf. J.W. LAPIERRE, Vivre sans État, Le Seuil, Paris, 1977. Cf. également Pierre BIRNBAUM, "Sur les origines de la domination politique (À propos d'Étienne de la Boétie et de Pierre Clastres)", in Revue française de science politique, $\mathrm{n}^{\circ} 27$ (1), 1977. Dans ce dernier article, Birnbaum attaque les positions de Clastres dont je parlerai plus loin, mais à tort je crois, car Clastres reconnait le conservatisme des sociétés primitives.

20. À propos de la crainte des dieux et de l'ordre qu'elle impose indépendamment d'un pouvoir politique étatique, of. Marcel GAUCHET, "La dette du sens et les racines de l'État ", in Libre 77 (2), Petite Bibliothèque Payot, Paris, et "Pierre Clastres " in Libre 78 (4). Selon ce dernier article, la société qui ne veut pas du pouvoir ne veut pas du changement. Ses membres veulent conserver la tradition parce qu'ils refusent à quiconque le droit de la changer. Voici comment Gauchet traduit leur pensée. "Personne parmi les hommes comme nous ne peut changer quelque chose à nos règles et à nos usages - c'est-a-dire exercer un pouvoir au sens proprement politique du mot. Car le pouvoir c'est toujours dans le fait de poser des lois nouvelles et de casser des lois anciennes qu'il consiste. Le pouvoir c'est d'abord le droit de redéfinir en permanence ce que doit être la société. Chose que justement la religion primitive décide impossible. Le conservatisme dicté par la croyance religieuse est ici le moyen de l'égalité politique. Car proclamer intangible la loi héritée, soustraire l'ordre collectif à la prise transformatrice des hommes, c'est empêcher le pouvoir en son ambition essentielle " (p, 62). 
volonté de tous. Dans une telle société, «faire le chef » est un manque de goût ou un crime qu'on ne tolère pas, qui peut être puni par la mort. Ce n'est pas assez dire qu'il y a là absence de pouvoir, il y a là "un refus institué et déterminé du pouvoir $^{21}$ ", "société contre l'État », selon la formule de Pierre Clastres.

Celui-ci va jusqu'à opposer deux sortes de chefs et de sociétés. Il y aurait, d'une part, des sociétés où le chef paie le privilège de jouer ce rôle et demeure toujours à la merci de son peuple. Il ne garde sa place qu'en gagnant sans cesse l'approbation de ses "sujets ", en prenant la responsabilité de les conduire comme ils le désirent, en distribuant tout ce qu'il a et en produisant plus que les autres de façon à pouvoir faire preuve de munificence ${ }^{22}$. D'autre part, il y a des sociétés où le chef assujettit son peuple, que ce soit par l'idéologie ou par la contrainte physique. Dans ce cas, c'est le peuple qui verse des prestations au chef (et à une éventuelle classe dominante). Ce n'est plus le chef qui doit des prestations à son peuple pour mériter son statut ${ }^{23}$. La différenciation des richesses et du pouvoir s'est alors établie dans la société de façon irrémédiable. Il y a désormais un maitre et des sujets, un État.

L'opposition établie par Clastres est limpide, mais trop simpliste pour rendre compte des données ethnographiques multiples. Je voudrais évoquer ici quelques cas qui semblent s'éclairer par des hypothèses qui, sans contredire celle de Clastres, la nuancent et lui conferent une portée plus large.

Jacques Dournes a décrit les institutions politiques des Jorai, ethnie de la péninsule indochinoise ${ }^{24}$. On trouve dans cette culture des maîtres apparemment prestigieux, richement

21. M. GAUCHET, ibid., p. 59.

22. Ce serait pour produire plus, que le chef aurait parfois droit à plus de femmes que ses "sujets ". Cf., à ce sujet, Claude Levi-Strauss, "La théorie du pouvoir dans une société primitive », in Les Dotrines politiques modernes, Brentano's Inc., New York, 1947.

23. Cf. la préface de Pierre Clastres au Livre de Marshall SAHLINS, op. cit.

24. "Sous le couvert des maitres", in Archives européennes de socinlogie, Tome XIV, n², 1973, p. 185-209. 
parés, dont le rôle est surtout de représenter leur peuple à l'extéricur, de traiter avec les royaumes étrangers de façon à forcer le respect de ceux-ci et à maintenir l'indépendance de leur peuple. Ces maitres ont aussi des fonctions magico-religieuses. Mais, en dehors de celles-ci et de la mise en scène en vue d'impressionner l'étranger, ils sont des paysans qui peinent aux champs comme n'importe lequel des Joraï.

Certaines royautés sacrées qu'on rencontre en Afrique ont été interprétées de façon aussi séduisante qu'audacieuse par René Girard ${ }^{25}$. Le roi remplirait la fonction bénéfique d'un bouc émissaire chargé des maux de son peuple. Il serait pour ce dernier une garantie contre les catastrophes, puisqu'il suffirait de le sacrifier pour se débarrasser des catastrophes ${ }^{26}$. Pour pouvoir garantir ainsi le salut public, le roi doit être chargé de toute opprobre. C'est ce qu'indiquent de nombreux rites d'intronisation où le roi effectivement transgresse les interdits les plus graves, accède à la royauté en même temps qu'à la solitude du réprouvé. Notez que ce que le roi porte seul sur ses épaules, c'est d'abord tout ce qui menace la concorde de son peuple. La catastrophe fondamentale, l'impiété originaire, c'est le désordre, le désordre social, l'absence de règles. Le roi commence donc son règne en brisant des règles fondamentales de la culture (en commettant rituellement un inceste, par exemple). S'il paraît au-dessus de ces règles, c'est qu'il a pour fonction de les restaurer en étant mis à mort.

Dans une étude sur les Rukuba (Plateau State, Nigeria), Jean-Claude Muller $^{27}$ suggère que bien des exactions des chefs Rukuba doivent s'interpréter non comme manifestation d'un pouvoir souverain, mais comme manifestation de la nature ambivalente du chef : pour être le bouc émissaire qui pourra sauver son peuple, il doit, à l'occasion et non seulement lors des rites d'intronisation, se rendre odieux. L'examen du mode de production et des prestations chez les Rukuba permet

\footnotetext{
25. Cf. La Violence et le sacré, Grasset, Paris, 1972, p. 150-166.

26. Ce sacrifice peut être accompli périodiquement, à titre préventif. Il peut n'être que symbolique ou bien épargner le roi tout en lui substituant une autre victime, humaine ou animale.

27. Cf. "Chefferie, idéologie et mode de production chez les Rukuba (Plateau State, Nigeria) ", in Revue canadienne des études africaines, XI (1), 1977, 3-22.
} 
d'affirmer que l'égalité économique des unités domestiques est entretenue et garantie par des usages culturels contraignants, qu'il n'y a pas de tribut proprement dit qui soit versé au chef. Quoique ce dernier soit le plus gros consommateur, il est aussi l'agent redistributeur des surplus. Il assure un nivellement des richesses par ses ponctions, mais lui-même ne peut que consommer ou redistribuer ce qui lui revient. Il ne peut jouir de ses privilèges et garder sa place que s'il assure la protection mystique des récoltes et de la paix du village. Si les récoltes sont mauvaises, ses greniers sont mis à la disposition des nécessiteux. Si elles sont bonnes, ils servent aux festivités qui assurent le bon renom du village.

On comprend difficilement qu'un tel chef puisse faire preuve d'arbitraire. Et pourtant il le fait, et de façon aussi voyante que scandaleuse. N'est-ce pas, dit Muller, parce qu'il doit se comporter comme celui qui se charge seul des crimes et des faillites de l'ordre social, celui qui pourra restaurer l'ordre social en étant sacrifié, de façon d'ailleurs très symbolique? Un tel chef ne l'est que selon l'idéologie qui définit et limite son rôle, idéologie par laquelle son peuple le maintient à son service $^{28}$. Il n'a aucun moyen d'exercer plus de pouvoir que celui qui lui est attribué conformément à cette idéologie. Celle-ci assure la prééminence du chef mais veille en même temps à ce que les prestations qu'il reçoit ne rompent pas l'égale répartition des moyens de production. Cette idéologie qui maintient le pouvoir politique dans des bornes strictes n'est ni une superstructure ni une conscience fausse au service d'une quelconque domination. Au contraire, c'est elle qui semble déterminer le fait que ne puisse émerger une classe dominante, un mode de production tributaire et un État.

Qu'en est-il de la prééminence des aînés dans les sociétés lignagères? Ne trouve-t-on pas là, en l'absence d'un État, une véritable exploitation d'une classe par une autre, si du moins on peut considérer que les aînés appartiennent à une même classe plutôt qu'à leur lignage respectif ? On a beaucoup écrit

28. P. CLASTRES semble d'accord avec ce point de vue comme avec celui de $J$. DOURNES. Le pouvoir formidable de certaines royautés africaines lui semble plus " apparent " que réel. Cf. "Liberté, malencontre et innomable ", in Le Discours de la servitude volontaire d'Étienne de la BOÉTIE, Payot, Paris, 1976, p. 234. 
sur cette question ${ }^{29}$. Il semble qu'on puisse dire que les aînés dominent leurs cadets mais ne les exploitent guère économiquement. S'il y a des exploités dans les sociétés lignagères, ce sont les femmes, et c'est l'ensemble des hommes qui profitent d'elles. Les aînés contrôlent la circulation de la main-d'œuvre et des épouses. Les cadets dépendent d'eux pour obtenir femmes et dépendants. Les prestations qu'ils versent aux aînés leur permettront donc d'améliorer leur statut. Certains deviendront à leur tour des aînés de lignage. Le contrôle exercé par les aînés porte essentiellement sur la reproduction des lignages (par segmentation ou fusion si nécessaire) en tant qu'unités de production viables et sensiblement égales entre elles, transigeant les unes avec les autres sans avoir à se soumettre à un maître commun.

Entre sociétés étatiques, où un tribut est versé par le peuple à ses maîttes, où le peuple est au service de ses maîtres, et sociétés sans État et sans tribut, où le mode de production est domestique ou lignager et n'implique aucune exploitation d'une unité productive par une autre, la distinction n'est pas toujours nette. Je viens d'évoquer quelques cas où il ne faut pas s'en tenir aux apparences. Sont-ce des cas intermédiaires? Entendons-nous bien. Ce n'est pas parce qu'on ne peut les ranger d'emblée dans un des deux types qu'oppose Clastres, qu'ils témoignent d'une transition entre ces deux types. D'autre part, les apparences d'un pouvoir étatique ou d'un tribut peuvent servir de couverture à une transformation subreprice de l'apparence en réalité, l'idéologie égalitaire qui reconnaît des chefs tout en les contrôlant de près, pourra être manipulée par ces chefs dans certaines circonstances.

Qu'est-ce qui pourrait bien amener un peuple où règne l'égalité à accepter des maîtres, à tolérer en son sein une différenciation de classe? On peut imaginer plusieurs hy-

29. Cf. Alain MARIE, "Rapport de parenté et rapport de production dans les sociétés lignagères ", in L'Antbropologie économique, édité par F. Pouillon, Maspero, Paris, 1976. Cet article cite une bibliographie importante sur la question. 
pothèses à défaut de pouvoir constater sur le vif la formation d'un pouvoir séparé de la société. D'abord, il y a la nécessité de conduire des guerres, et l'on envisage facilement qu'un leader militaire fasse durer les hostilités (ou la menace des hostilités) pour garder toute l'importance que celles-ci conferent à sa fonction et pour garder des forces armées à sa disposition. On peut aussi imaginer qu'un chef profite du commerce extérieur ou d'un monopole quelconque qui s'attacherait à sa fonction pour gagner du pouvoir. Par exemple, il peut devenir l'interlocuteur privilégié, puis le commis attitré, d'une puissance coloniale ${ }^{30}$. La croissance démographique de la société entraîne éventuellement l'émergence de chefs dont le pouvoir deviendrait de plus en plus indépendant du consentement de tous. Car il semble que seuls des groupes restreints soient capables de se diriger eux-mêmes sans se confier à des maîtres ${ }^{31}$.

Ce ne sont là que quelques hypothèses. Mais elles ont toutes un caractère commun : elles mettent en jeu un facteur extérieur. Une société sans État n'a en effet aucune raison endogène de renoncer à l'égalité et à l'absence d'asservissement $^{32}$.

30. En Afrique de l'Ouest, les aînés de lignage jouissaient d'un ascendant certain et recevaient des prestations de leurs cadets, mais ils les redistribuaient en vue d'établir leurs cadets et de perpécuer un mode de production où les lignages demeuraient des unités sensiblement égales. Le commerce des esclaves permettait de maintenir un certain équilibre démographique entre lignages. Cependant, avec la traite et la demande extérieure d'esclaves, l'équilibre se brisa. Sur la côte, des royaumes apparaissent en même temps que l'inégalité s'installe entre lignages, les uns devenant de plus en plus forts, profitant du commerce et faisant le profit des armateurs. (Cf. notamment P. P. REY, "Articulation des modes de dépendance et des modes de production dans deux sociétés lignagères " in Cabiers d"études africaines, IX, 35, p. 415-440 et R. HORTON, "From Fishing Village to City-State [A social history of New Calabar]", in Man in Africa, édité par M. Douglas et P. M. Kaberry, Tavistock Publications, Londres, 1969.)

31. Évidemment, les groupes peuvent se fissionner, quand ils deviennent trop gros, et se multiplier. Une population nombreuse et de même culture peut ainsi s'émietter en une poussière de petites communautés, souveraines et acéphales, si celles-ci trouvent l'espace et les ressources voulues pour se tenir à distance les unes des autres. Vu de l'extérieur, on ne percevra qu'une même société, mais il s'agira en fait d'une multiplicité de groupes souverains s'opposant les uns aux autres. Cf. P. CLASTRES, «Archéologie de la violence ", in Libre, 77 (2), Payot, Paris.

32. L'État semble bien être le fruit d'une série de circonstances qui provoqueraient l'apparition d'une organisation centralisée du pouvoir et l'acceptation de cette nouvelle organisation au nom même d’une idéologie de la réciprocité. Petit à petit, un tribut régulier serait établi, la possibilité de fission s'amenuiserait, des classes sociales se différencieraient, mais ce sont là des résultats, non des causes de l'émergence de l'État. Des structures traditionnelles servent sans doute de support au nouveau pouvoir (cf. $\mathbf{R}$ 
Pierre Clastres a insisté sur ce dernier point ${ }^{33}$. Dans une telle société, l'économie est subordonnée aux besoins d'hommes égaux et qui entendent le demeurer. Personne n'y a le pouvoir de contraindre autrui. Et personne ne peut acquérir un tel pouvoir s'il n'existe aucun appareil de contrainte idéologique ou politique. Sans cet appareil, il ne peut se produire une différenciation des classes et donc ce n'est pas cette différenciation qui peut rendre compte de l'apparition de cet appareil. En d'autres mots, l'idéologie de l'égalité est déterminante et non déterminée dans une société sans État. Encore faut-il expliquer ce que cela signifie.

Rapports de production, rapports de domination et idéologie sont trois faces d'une même réalité. Un système de pouvoir ou d'exploitation ne va pas sans une certaine logique des représentations. Un ordre social s'institue à la fois dans la tête des gens, dans la distribution des statuts et dans la division sociale du travail. Il ne peut changer qu'en changeant sur tous ces tableaux, quoique pas nécessairement en même temps ni au même rythme ${ }^{34}$. Ceci dit, on conviendra que, dans certaines sociétés, ce soit l'organisation du travail qui joue le rôle dominant, dans d'autres, ce sera la contrainte politique ou le discours idéologique. Et d'ailleurs, hors des sociétés capitalistes, la distinction de ces trois instances n'est pas toujours éclairante.

J'ai envisagé des sociétés sans État où le discours idéologique n'est pas tenu au profit de maîtres ou d'exploiteurs, où ceux-ci et ceux-là n'existent pas ${ }^{35}$. Par contre, ce

HORTON, "Stateless Societies in the History of West Africa", Vol. I. édité par J.F.A. Ajayi et M. Crowder, Londres, Longman, 1971), mais ce nouveau pouvoir transforme les structures traditionnelles. L'émergence de l'État est un phénomène long et complexe qui entraine divers effets secondaires. Ceux-ci deviennent à leur tour des causes déterminantes. Il est ridicule de voir dans cette affaire la conspiration d'une même classe. Tout au long de ce processus, les clivages sociaux, les mentalités et les intérêts se modifient de fond en comble. (Cf. The Early State, édité par H.J.M. Claessen et P. Skalník, La Haye, Mouton, 1978. Cf. notamment les chapitres écrits par R. Cohen et les éditeurs.)

33. Cf. le chapitre 11 dans La Société contre l'État. op. cit.

34. La dialectique entre ce qui est déjà institué et ce qui s'institue se traduit par une dialectique entre idées reçues, politiques et économie. Dans chacun de ces registres, les inerties cle ce qui est déjà institué sont différentes.

35. Encore quil faille ne pas oublier le sort des femmes et des cadets. 
discours demeure inséparable d'un ordre social institué, d'une économie de subsistance et d'un contrôle de tout leader par ses compagnons ${ }^{36}$. Si un leader se transforme en maître, si un aîné s'impose aux aînés des autres lignages, si un tribut lui est réellement versé, cela ne pourra se produire que par manipulation de l'idéologie. Celui qui pourra se porter garant de l'" ordre des choses" ou de la "volonté des ancêtres", à l'occasion de circonstances neuves, celui-là pourra éventuellement acquérir du pouvoir sur ses compagnons. On imagine facilement comment un guerrier valeureux ou un individu en relation avec les esprits peuvent devenir, dans une situation de crise, prophètes millénaristes ou nativistes, comment en prêchant la sauvegarde d'une identité culturelle, en luttant pour la liberté, ils en viennent à asservir leur communauté.

\section{Cégep de Saint-Laurent}

36. Aussi peut-on relancer le débat. L'idéologie, si elle est dominante dans de telles circonstances, n'est-elle pas elle-même déterminée à jouter les premiers rôles par les conditions matériclles de l'existence? Cependant, il faut bien remarquer que celles-ci ne constituent pas une détermination ultime, si ce n'est de façon négative, dans les sociétés précapitalistes. Dans ces sociétés, les conditions matérielles n'autorisent pas n'importe quelle forme de culture, mais n'impliquent pas pour autant une forme particulière. L'écologiest les techniques ne donnent lieu à tel ou tel mode de production qu'en étant mises en oeuvre dans des rapports de production et ceux-ci sont inséparables des rapports de pouvoir (ou d'égalité) et d'une idéologie. (Cf. A. MARIE, op. cit., p. 115-116.) 